\title{
Profilaxis antimicrobiana en otitis media recurrente
}

\author{
JACOB COHEN V.
}

Chemoprophylaxis of recurrent otitis media

Palabras claves: otitis media recurrente; profilaxis; vacunas; cirugía.

Key words: Recurrent otitis media; prophylaxis; vaccines; surgery.

\section{Introducción}

La otitis media aguda (OMA) en niños, es la afección más frecuentemente involucrada en la indicación de un antimicrobiano en países desarrollados como E.U.A ${ }^{1}$. El costo estimado del manejo de esta patología, en ese país, durante el año 1995 fue de 1,96 billones de dólares ${ }^{2}$. Durante el año 1999 se efectuaron 30 millones de visitas médicas por este concepto con 809 prescripciones de antimicrobianos por 1.000 visitas $^{3}$.

En la evolución clínica de la patología infecciosa del oído medio hay una serie de circunstancias en que ésta puede ser recurrente o persistente, con efusión o crónica, supurativa o sin supuración y en tales situaciones se plantean diferentes alternativas de manejo médico, quirúrgico o terapia combinada; una de estas discutidas alternativas es la quimioprofilaxis.

\section{Conceptos generales sobre profilaxis antimicrobiana}

La antibioprofilaxis suele indicarse en diferentes y muy variadas situaciones clínico-epidemiológicas. En pediatría, la administración de estos medicamentos para prevenir infecciones específicas o inespecíficas ha demostrado una eficacia inconstante según las diferentes variables en curso. Hay muy pocas indicaciones de profilaxis indiscutibles; en la mayoría de las prácticas no hay una base demostrada para utilizarlos ${ }^{4}$.

La quimioprofilaxis está orientada en niños hacia diversos objetivos que no se excluyen entre sí, a saber:

- A patógenos específicos. Ej. Bordetella pertussis, Neisseria meningitidis, Pneumocystis jiroveci.
- A zonas anatómicas con predisposición a infecciones. Ej. oído medio, tracto urinario, válvulas cardíacas, herida quirúrgica.

- A pacientes vulnerables. Ej. Recién nacidos, desnutridos, inmunosuprimidos, pacientes sometidos a trasplantes, pacientes con VIH/ SIDA, diabéticos.

Las indicaciones más eficaces de profilaxis se logran especialmente en el caso de patógenos específicos y/o en aquellas dirigidas a infecciones en determinadas zonas anatómicas. Cualquier sea la situación clínica en la que se considere una intervención de este tipo, debe sopesarse el riesgo a mediano o largo plazo de la presión selectiva ejercida por los antibacterianos sobre la microbiota y la selección de microorganismos multiresistentes versus los posibles beneficios preventivos.

En términos generales la profilaxis antimicrobiana debe tener el espectro de acción más reducido posible y llevarse a cabo sólo durante el tiempo que haya sido demostrado como mínimo necesario.

La prevención de las infecciones en áreas anatómicas específicas, como es el caso del oído medio, se ha discutido y planteado cuando el período de riesgo está más o menos definido, por períodos breves, los microorganismos patógenos esperados poseen una susceptibilidad in vitro previsible y la concentración del antimicrobiano a elegir, sea la más adecuada.

\section{Definición del problema clínico}

En la actualidad existen controversias en el manejo clínico de la otitis media (OMA), como

Clínica Las Condes, Departamento de Pediatría, Facultad de Medicina Universidad de Chile. Departamento de Pediatría Oriente-Hospital Luis Calvo Mackenna, Santiago, Chile. 
es la definición de la utilidad de los antimicrobianos, en la etapa aguda y no complicada. La recomendación de la Academia Americana de Pediatría, en el año 2004 consiste en manejo sintomático del dolor y de las manifestaciones generales como fiebre, durante las primeras 48 a $72 \mathrm{hrs}$, absteniéndose de usar antimicrobianos ${ }^{5}$. Posteriormente, bajo control médico y según sea la evolución, se decidirá si es necesario agregar una terapia antibacteriana, que de elección debe ser amoxicilina.

En porcentajes que fluctúan entre 20 y $25 \%$ según la casuística, los niños bajo dos años de edad presentan entre 3 y 5 episodios de OMA. La otitis media recurrente (OMR) se define como tres o más episodios de OMA en seis meses o más de cuatro en un año ${ }^{6}$.

El creciente problema de la resistencia de Streptococcus pneumoniae a penicilina y la conocida de Haemophilus influenzae y Moraxella catarrhalis a ampicilina, ha sido invocado como una de las causas de fracaso terapéutico y de las consecuentes recurrencias a corto plazo $^{7}$. Sin embargo, existen numerables factores de riesgo para la OMR, muchos de ellos controlables, con lo cual se disminuyen sustancialmente los períodos de recurrencia (Tabla 1 y Figura 1).

\section{Microbiología de la OMR}

Los microorganismos involucrados en OMR, son los mismos que en OMA: S. pneumoniae, $H$. influenzae y $M$. catarrhalis ${ }^{7}$. Uno de los problemas emergentes más significativos es la creciente resistencia a los antimicrobianos $\beta$-lactámicos por parte de $S$. pneumoniae. La colonización faríngea por $S$. pneumoniae es significativamente

Tabla 1. Factores de riesgo para adquirir una otitis media recurrente

Edad: $<$ de 3 años

Prematurez

Sexo masculino

Etnia: anglosajones y nativos americanos

Genéticos: predisposición familiar

Lactancia artificial (alimentación en posición decúbito)

Padres fumadores

Uso prolongado de chupete de entretención

Asistencia a guardería infantil

Nacimiento en otoño

Alergias respiratorias en la familia

Nivel socio económico bajo más prolongada en niños que presentan OMR que niños sin OMR, posiblemente porque el uso reiterado de antimicrobianos disminuye sustancialmente la colonización faríngea por Streptoccocus alfa hemolíticos, el que antagonizaría, compitiendo por receptores con $S$. pneumoniae . $^{8}$.

Por otro lado, en muchos casos, la microbiología de las recurrencia se presenta con cultivos aeróbicos negativos, debido en gran parte, a que los tratamientos prolongados con antimicrobianos efectivos erradican el agente causal, pero dado la evolución prolongada que ha tenido la recurrencia, los signos y síntomas persisten, porque la inflamación persiste.

\section{Estrategias de prevención en OMR}

Se analizan a continuación las diferentes alternativas planteadas para prevención de recurrencias de infecciones del oído medio, entendidas como un complemento a los esfuerzos orientados a evitar aquellos factores de riesgo evitables entre los enunciados en la Tabla 1.

\section{Quimioprofilaxis}

Desde la década del 70' numerosas experiencias clínicas mostraban algún beneficio con el uso de antimicrobianos administrados en una dosis diaria y nocturna por tiempos variables, hasta uno o dos años, en comparación con la evolución natural o la intervención quirúrgica. Maynaud y cols, en un estudio prospectivo y randomizado comparando amoxicilina $20 \mathrm{mg} / \mathrm{kg}$

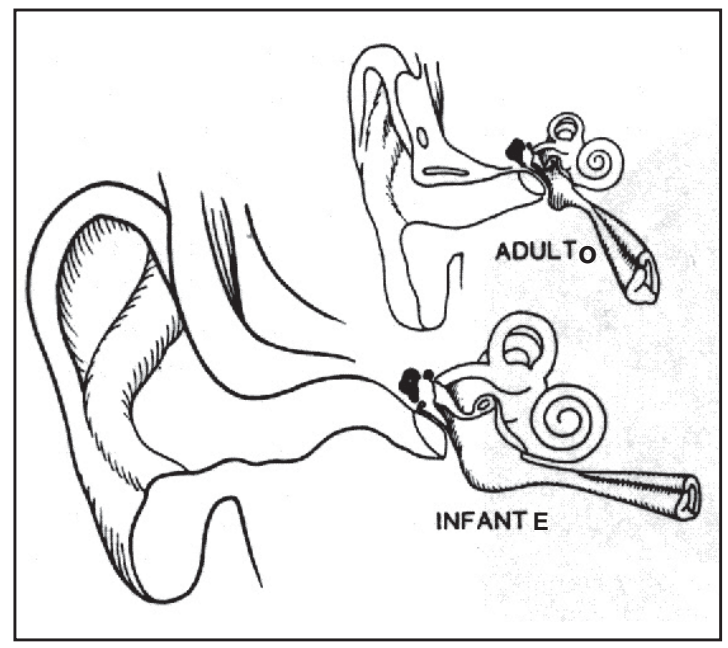

Figura 1. La diferente posición, tamaño, diámetro del lumen interno e inclinación de la Trompa de Eustaquio en el niño, favorece el reflujo de contenido faríngeo hacia el oído medio aumentando el riesgo de infección. 
día durante 2 años versus tubos de ventilación o placebo, en niños menores de 3 años con historia de OMR, demostraron que ambas intervenciones reducían de manera estadísticamente significativa la frecuencia, duración y severidad de nuevos episodios ${ }^{9}$. Otros grupos reportaron que antibacterianos como eritromicina/sulfisoxazol, cotrimoxazol o cefalosporinas orales de $2^{\mathrm{a}}$ generación, en ensayos clínicos dobles ciegos, prospectivos y comparativos, eran significativamente efectivos, hasta un año de observación, en la prevención de recurrencias ${ }^{10}$.

En la década de los 90' se comenzó a evidenciar, in vitro y en ensayos clínicos, que el uso prolongado de antimicrobianos del tipo $\beta$ lactámicos en bajas dosis como profiláctico, se asocia fuertemente con la aparición de resistencia, especialmente de $S$. pneumoniae, y en concomitancia se comenzó a observar mayor fracaso de los diferentes esquemas preventivos y mayor riesgo de infecciones óticas de más difícil manejo $^{11,12}$

Un meta-análisis sobre profilaxis antimicrobiana en OMR efectuado por Chalmers y cols en el año 1993, demostró que diferentes esquemas como sufisoxazol, cotrimoxazol o amoxicilina resultan en una ventaja, al disminuir en 0,11 episodios por niño y por mes, o cerca de un episodio de OMA por año, en estos pacientes ${ }^{13}$. Este pequeño beneficio se contrapone con la fuerte presión selectiva en la promoción de resistencia.

\section{Cirugía otorrinolaringológica}

Medidas como la instalación de tubos de ventilación, amigdalectomía y/o adenoidectomía solas o combinadas, demuestran escasos beneficios, con tan solo una disminución en 0,7 episodios por niño por año, en tres años de seguimiento ${ }^{6}$.

\section{Vacunas}

La evaluación de vacunas ${ }^{14,15}$ en la prevención de recurrencias ha demostrado que, específicamente la anti neumocóccica conjugada 7 valente, otorga una disminución en la incidencia de OMA de $6 \%$ global y de $60 \%$ para los serotipos vaccinales; la vacuna anti $H$. influenzae tipo b produce una reducción menor, sólo $3 \%$; por último, la vacunación anti influenza es la que mejores resultados epidemiológicos ha demostrado $^{1,14}$, reduciendo la frecuencia de OMR en 30 a $36 \%$ (la OMA es facilitada por las epidemias de virus infuenza).

\section{Comentario}

A pesar del fuerte impacto en salud pública y en la dificultad que ofrece el manejo clínicoepidemiológico, las diferentes medidas aplicadas no han demostrado tener resultados muy halagadores para las infecciones recurrentes del oído medio. Nuestros esfuerzos deberían estar dirigidos a intervenir dirigidamente en el control de los factores de mayor riesgo:

- Fomentando la lactancia materna.

- Revisando la técnica de administración del biberón.

- Eliminando en lo posible el chupete de entretención.

- Educando a los padres sobre la contaminación ambiental que producen en el hogar algunos calefactores y la necesidad de ventilar los ambientes, en especial en meses fríos.

- Censurar los hábitos de tabaquismo familiar.

- Evaluar la existencia de alergia respiratoria si existen antecedentes familiares al respecto.

- Sospechar oportunamente la existencia de defectos inmunológicos como defectos de inmunoglobulinas séricas o de fagocitosis, que si bien son muy infrecuentes, pueden debutar como OMR en lactantes pequeños.

- En algunos pacientes llega a ser necesario que el pediatra recomiende el retiro del niño de guardería infantil si la OMR parece insoluble.

En contados casos se podría plantear la cirugía, como son los tubos de ventilación uni o bilaterales y/o la adenoidectomía.

Respecto a vacunas se ha planteado que una vacunación anti influenza anual y oportuna podría disminuir la incidencia de OMA en general y de OMR por consecuencia.

Evaluaciones sistemáticas de las vacunas anti $H$. influenzae b y anti neumocóccica (tanto conjugada como polisacarídica) no han podido demostrar un beneficio significativo en la prevención global de OMR y por tanto, no debiera indicarse estas vacunas con este objetivo.

Ha quedado bien demostrado que no se deben usar antimicrobianos profilácticos en esta patología, por el escaso beneficio alcanzado y por la enorme presión selectiva que se ejerce sobre la aparición de cepas multiresistentes tanto en quienes reciben la profilaxis como en el resto de la comunidad.

\section{Bibliografía}

1.- Arrieta A, MD, Singh J, MD. Management of recurrent and persistent acute otitis media: new options with 
familiar antibiotics. Pediatr Infect Dis J 2004; 23: S115-24.

2.- Blumer J L. Fundamental basis for rational therapeutics in acute otitis media. Pediatr Infect Dis J 2000; 19: 911-16.

3.- McCaig L F, Besser R E, Hughes J M. Trends in antimicrobial prescribing rates for children and adolescents. JAMA 2002; 287: 3096-102.

4.- Report of the Committee on Infectious Diseases: Section 5 Antimicrobial Prophylaxis, Pickering Larry K. M.D. Red Book; $26^{\text {th }}$ edition; Elk Grove Village, IL. American Academy of Pediatrics; 2003: 729-80.

5.- Subcommittee on Management of Acute Otitis Media, American Academy of Pediatrics. Diagnosis and Managements of Acute Otitis Media. Pediatrics 2004; 113: 1451-65.

6.- Bailey Byron J. Head \& Neck Surgery- Otolaryngology Third ed. Vol. One Ed. Lippincott Williams \& Wilkins 2001. Bluestone Charles cap. 82 Controversies in Tonsillectomy, Adenoidectomy and Tympanostomy tubes: Recurrent Acute Otitis Media pag. 999-1005.

7.- Leibovitz E, Greenberg D, Piglansky L, Raiz S, Porat $\mathrm{N}$, Press J, et al. Recurrent acute otitis media occurring within one month from completion of antibiotic therapy: relationship to the original pathogen. Pediatr Infect Dis J 2003; 22: 209-16.
8.- Pichichero M E. Recurrent and persistent otitis media. Pediatr Infect Dis J 2000; 19: 911-16.

9.- Maynaud J E, Fleshman J K, Tschoff C F. Otitis media in alaskan eskimo children: prospective evaluation of chemoprophylaxis JAMA 1972; 219: 597-9.

10.- Perrin J M, Charney E, MacWhinney J B Jr, McInerny T K, Miller R L, Nazarian L F. Sulfisoxazole as chemoprophylaxis for recurrent otitis media: a doubleblind crossover study in pediatric practice. $\mathrm{N}$ Engl $\mathrm{J}$ Med 1974; 291: 664-7.

11.- Hendley J O. Otitis media. N Engl J Med 2002; 347: 1169-74.

12.- Amsden G, Pharmd F C O. Pneumococcal resistance in perspective: how well are we combating it? Pediatr Infect Dis J, 2004; 23: S125-8.

13.- Williams R L, Chalmer T C, Stange K C, Chalmers F T, Bowlin S J. Use of antibiotics in preventing recurrent otitis media and in treating otitis media with effusion: a meta-analytic attempt to resolve the brouhaha. JAMA 1993; 270: 1344-51.

14.- Greenberg D P, Hoberman A. Vaccine prevention of acute otitis media. Curr Allergy Asthma Res 2001; 1: 1948-54.

15.- Fireman B, Black S B, Shinefield H R, Lee J, Lewis E, Ray P. Impact of the pneumococcal conjugate vaccine on otitis media. Pediatr Infect Dis J 2003; 22: 10-6.

Correspondencia a:

Jacob Cohen Ventura

jcohen@clinicalascondes.cl 